\title{
ANÁLISES MORFOMÉTRICAS ENTRE APIS MELLIFERA DA MESORREGIÃO DO SERTÃO PARAIBANO*
}

\author{
MORPHOMETRIC ANALYSES BETWEEN APIS MELLIFERA \\ FROM REGION SERTÃO PARAIBA STATE, BRAZIL
}

\author{
Souza, D.L. ${ }^{1}$, A. Evangelista-Rodrigues ${ }^{2}$, M.N. Ribeiro ${ }^{3}$, F. Padilla Álvarez ${ }^{4}$, E.S.L. Farias ${ }^{5}$ \\ e W.E. Pereira ${ }^{2}$

\begin{abstract}
${ }^{1}$ Centro de Ciências Agrárias-UFPB. Areia. Paraíba. Brasil. UFPB. Areia. Paraíba. 58397-000. Brasil. adriana@cca.ufpb.br.

${ }^{3}$ Departamento de Zootecnia UFRPE. Recife. Pernambuco. Brasil. ${ }^{4}$ Departamento de Zoologia. Universidad de Córdoba. Córdoba. España.
\end{abstract} \\ ${ }^{2}$ Centro de Ciências Agrárias-UFPB. Departamento de Zootecnia. Setor de Apicultura. Campus II. CCA/ \\ ${ }^{5}$ Graduando do Curso de Zootecnia CCA/UFPB. Areia. Paraíba. Brasil.
}

\section{PALAVRAS CHAVE ADICIONAIS}

Abelha. Asa. Fêmur. Tibia. Tarso. Probóscide.

\section{RESUMO}

Objetivou-se, com esse trabalho, caracterizar as populações de Apis mellifera da mesorregião do Sertão paraibano, comparando os indivíduos morfometricamente. As medidas tomadas foram: comprimento da asa anterior (CAA) e largura da asa anterior (LAA), ângulos B4, E9, G18, J16, K19, N23, O26 e distância b (Db); comprimento da asa posterior (CAP) e largura da asa posterior (LAP), distância L2 (DL2), L5 (DL5) e ângulo W3, comprimento do fêmur (CF), comprimento da tíbia (CT), comprimento do tarso (CTS) e largura do tarso (LTS) e comprimento da probóscide (CP). Para a comparação das diferentes variáveis estudadas utilizou-se a análise de variância, o teste de Tukey, análise canônica e o teste de Tocher a $5 \%$ de significância. Para as medidas realizadas em cada amostra utilizou-se um equipamento de análise de imagens, Programa Image Pro Plus 4.0. As variáveis CTS e LTS, ângulos G18 e K19 e DL2 diferiram significativamente $(p<0,05)$ entre as localidades estudadas de acordo com o teste de Tukey. A análise canônica mostrou a existência de um agrupamento com base nas características morfológicas entre duas das localidades estudadas apesar da distância geográfica. Concluiu-se que existem dois grupos

*Parte da dissertação do primeiro autor.

Recibido: 11-12-06. Aceptado: 17-1-08.

\section{Additional KeYWORDS}

Honeybees. Wing. Femur. Tibia. Tarsus. Proboscis.

distintos morfometricamente para a microrregião estudada e que há um processo adaptativo das abelhas, quanto às medidas morfométricas, para as diferentes regiões geomorfológicas.

\section{SUMMARY}

This research was carried out to characterize Apis mellifera populations from region Sertão from Paraiba state, comparing the individual's morphometry. The parameters measured were: forewing length (FwL) and forewing width (FwW), B4, E9, G18, J16, K19, N23, O26 angles, and b distance $(\mathrm{Db})$; hindwing length $(\mathrm{HL})$ and hindwing width (HW), L2 (DL2), L5 distance (DL5) and W3 angle, femur lengths (FL) and tibia lengths (TL), tarsus length (TL) and tarsus width (TW) and proboscis length $(\mathrm{PL})$. To compare different variables, a data united analysis (ANOVA), Tukey's and Tocher's test to $5 \%$ of significance. For the measures accomplished in each sample an equipment of analysis of images, and Program Image Pro Plus 4.0 were used. The variables TsL and TsW, G18 and K19 angles and DL2 distance presented significant difference $(p<0.05)$ among the places studied by Tukey's test. Canonical discriminant analysis showed a grouping with base in the morphologic characteristics between 
two places studied in spite of the geographical distance. It was concluded there are two morphometric different groups for studied hinterland region. There is an adaptation of the bees, with relationship to morphometric measures, for different geomorphologic regions.

\section{INTRODUÇÃO}

O Brasil apresenta clima tropical, extensa área territorial e vegetação diversificada o que vem a favorecer a atividade apícola que associada às novas técnicas aplicadas representa um potencial para a exploração da atividade. O estado da Paraíba localizase na região semi-árida nordestina, possui uma vegetação predominantemente de caatinga, a qual apresenta uma flora bem diversificada e rica em néctar e pólen, oferecendo alimento para as abelhas nas diversas épocas do ano.

O Sertão paraibano divide-se em sete microrregiões devido ao maior ou menor grau de umidade que caracteriza suas diversas áreas com clima variando de quente e seco a temperado. Possuem vegetação típica de caatinga primitiva arbustiva esparsa, sendo a caatinga um dos maiores ecossistemas brasileiros e ocupando uma grande parte da área territorial do Nordeste com uma fauna apresentando propriedades diversas que lhes permitem viver em condições adversas. Tendo como principais espécies vegetais: marmeleiro, pereiro, jurema, angico, dentre outras (Maia, 2004; Carvalho etal., 2002a).

As abelhas são essencialmente vespas que abandonaram a depredação em favor do aprovisionamento de seus ninhos com néctar e pólen. Acredita-se que as abelhas, tendo como antepassada a vespa, evoluíram seu aparelho bucal para serem capazes de ingerir néctar, em vez de caçar, começaram a coletar pólen para alimentar sua cria (Winston, 2003). As famílias dos Apóideos se caracterizam por estruturas minuciosas. Nutrem-se com néctar e pólen das flores, de onde retiram carboidratos e proteínas. De um modo geral, as espécies sociais se diferenciam das solitárias e das parasitas pela conformação das pernas posteriores (Carrera, 1980).

$\mathrm{Na}$ apicultura as pesquisas estão em uma posição privilegiada, desde que é sabido que as raças geográficas, corretamente definidas, têm individualidade biológica. Os problemas verdadeiros, entretanto, são encontrados nos grupos de raças existentes há muito tempo com similaridade morfométricas e na variabilidade dentro das subespécies (Ruttner, 1988).

$\mathrm{O}$ aspecto mais importante da biologia da abelha é a relação entre a forma e função. Os aspectos morfológicos têm sido muito empregados na caracterização das diferentes raças de abelhas, (Ruttner, 1988). Visto que a forma e tamanho da cesta de pólen tem correlação com a produção de mel da colmeia, o comprimento e largura da asa está diretamente relacionada com a capacidade de vôo da abelha e a maior ou menor entrada de néctar na colméia tem haver com o comprimento da probóscide e o tipo de corola da flor visitada pela abelha. Estudos realizados por Sereno et al. (2002) concluíram que a utilização de médias de caracteres morfológicos por colmeia permite comparar diferentes grupos de abelhas, existindo diferenças regionais entre colmeias, como citado por Diniz-Filho et al. (2000) incluindo o conhecimento sobre a herdabilidade destes caracteres. Falconer (1989) estudando os valores dependentes das medidas de qualidade tanto quanto de quantidade, encontrou uma correlação entre o genótipo e o fenótipo, que é modificada pelos efeitos ambientais. No entanto, a herdabilidade das características morfológicas, diminui quando as abelhas são criadas sob condições alimentares adversas. Diante desse exposto, objetivou-se, com esse trabalho, caracterizar as populações de Apis mellifera da microrregião do Sertão paraibano, comparando os indivíduos morfometricamente. 


\section{MATERIALE MÉTODOS}

As amostras foram coletadas no período de março a setembro de 2005 nos municípios de: Catolé do Rocha $\left(06^{\circ} 57^{\prime} 46^{\prime \prime} \mathrm{S}, 35^{\circ} 41^{\prime} 31^{\prime \prime} \mathrm{O}\right)$, Patos $\left(07^{\circ} 01^{\prime} 28^{\prime \prime S}\right.$, $\left.37^{\circ} 16^{\prime} 48^{\prime \prime O}\right)$ e Princesa Isabel $\left(07^{\circ} 44^{\prime} 12^{\prime \prime} \mathrm{S}, 38^{\circ} 59^{\prime} 36^{\prime \prime} \mathrm{O}\right)$ por possuírem os apiários mais representativos. Os municípios de Catolé do Rocha e Princesa Isabel caracterizam-se pela presença das unidades geomorfológicas denominadas de Borborema e Serras, enquanto que Patos caracteriza-se por se encontrar em um pediplano sertanejo com inselbergs (morros isolados) (Carvalho et al. (2002b).

De cada município foram coletadas amostras (abelhas) aleatórias de apiários fixos formados de colônias naturais, com colmeias modelo Langstroth e conservadas em álcool a $70 \%$. De cada amostra foram confeccionadas 30 lâminas de acordo com a metodologia descrita por Ruttner (1988).

As medidas analisadas foram sobre as asas anterior e posterior do lado direito da abelha, a terceira perna do lado direito e a probóscide, sendo as seguintes: comprimento da asa anterior (CAA) e largura da asa anterior (LAA); ângulos da asa anterior B4, E9, G18, J16, K19, N23, O26 e distância $\mathrm{b}(\mathrm{Db})$; comprimento da asa posterior (CAP) e largura da asa posterior (LAP), distância L2 (DL2), L5 (DL5) e ângulo W3; comprimento do fêmur (CF); comprimento da tíbia (CT); comprimento do tarso (CTS) e largura do tarso (LTS) e comprimento da probóscide (CP). Cada lâmina foi colocada na platina do estereoscópio sobre uma folha de papel milimetrado, em uma posição horizontal. A imagem visualizada mostrou uma leitura correspondente da peça sobre a imagem de uma objetiva ocular de 10x num aumento micrométrico de 1,5. Para as medidas realizadas em cada amostra utilizou-se um equipamento de análise de imagens, Programa Image Pro Plus 4.0.

Os dados foram analisados estatisticamente por meio de análise de variância (ANOVA). A análise canônica foi realizada para testar o efeito de região considerando todas as características avaliadas de forma simultânea e os escores foram submetidos à análise de agrupamento. Foram utilizados o teste de Tocher e o teste de Mahalanobis como métodos de agrupamento que utilizam a distância euclidiana como medida de dissimilaridade entre duas comunidades, ao nível de 5\% de significância analisados estatisticamente com a ajuda do programa SAEG 5.0(1999).

\section{RESULTADOSEDISCUSSÃO}

As variáveis: $\mathrm{CP}$; $\mathrm{CF}$; Db; ângulos B4, E9, J16, N23, O26; LAP; DL5 e ângulo W3 não apresentaram diferenças significativas ao nível de 5\%. A variável DL5, presente na asa posterior apresentou um coeficiente de variação de $119,89 \%$, apesar de não ter diferido significativamente. Isto demonstra uma variação entre os valores médios máximos e mínimos para a distância L5 de 10 vezes $(0,06-0,96)$. Ao extrapolar estes valores para as regiões trabalhadas pode-se interpretar que, sendo as venações características adaptativas utilizadas para o estudo genético e taxonômico, houve uma adaptação bastante regionalizada. Ken et al. (2003) estudando as características morfológicas de Apis cerana provenientes da Província de Yunnan, China, usando 38 caracteres morfométricos encontraram resultados que mostraram um alto grau de variação o qual correlatou a parâmetros geográficos.

Ruttner (1988), afirma que os ângulos das venações são independentes do tamanho da asa, e mostram a variação geográfica significativa; isto significa que reage sensivelmente aos processos de evolução, mesmo ao nível de subespécies. O mesmo autor cita que as venações da asa da abelha doméstica provaram ser fontes ricas para análise genética e taxonômica e que através delas pode-se medir exatamente, o grau de herdabilidade.

A tabela I mostra os resultados do teste de Tukey para as variáveis morfométricas 
Tabela I. Resultados do teste de Tukey aplicado as 20 variáveis morfológicas (comprimento, largura e distância em mm e ângulos em graus) analisados nas abelhas Apis mellifera da mesorregião do Sertão paraibano. (Results of applied Tukey test to the 20 morphologic variables (length, width and distance in $\mathrm{mm}$ and angles in degrees) analyzed in the bees Apis mellifera of the region from Sertão paraibano).

\begin{tabular}{|c|c|c|c|c|c|}
\hline \multirow[t]{2}{*}{ Variáveis } & \multicolumn{4}{|c|}{ Localidades (médias em mm) } & \multirow[b]{2}{*}{ Média geral (mm) } \\
\hline & $\mathrm{N}$ & Catolé do Rocha & Princesa Isabel & Patos & \\
\hline $\mathrm{CP}$ & 90 & $4,85^{\mathrm{a}}$ & $4,85^{\mathrm{a}}$ & $4,74^{\mathrm{a}}$ & $4,81 \pm 0,40$ \\
\hline $\mathrm{CF}$ & 90 & $2,35^{\mathrm{a}}$ & $2,39^{a}$ & $2,37^{a}$ & $2,37 \pm 0,09$ \\
\hline СТ & 90 & $2,92^{\mathrm{a}}$ & $2,97^{a}$ & $2,94^{b}$ & $2,91 \pm 0,10$ \\
\hline CTs & 90 & $1,83^{b}$ & $1,95^{\mathrm{a}}$ & $1,89^{a b}$ & $1,89 \pm 0,11$ \\
\hline LTs & 90 & $1,17^{\mathrm{ab}}$ & $1,19^{a}$ & $1,16^{b}$ & $1,17 \pm 0,05$ \\
\hline CAA & 90 & $8,34^{b}$ & $8,45^{a}$ & $8,31^{b}$ & $8,37 \pm 0,18$ \\
\hline LAA & 90 & $2,92^{b}$ & $2,98^{a}$ & $2,93^{b}$ & $2,95 \pm 0,07$ \\
\hline $\mathrm{Db}$ & 90 & $0,24^{\mathrm{a}}$ & $0,24^{a}$ & $0,23^{a}$ & $0,23 \pm 0,03$ \\
\hline Ângulo B4 & 90 & $105,61^{a}$ & $103,13^{a}$ & $102,87^{a}$ & $103,88 \pm 6,77$ \\
\hline Ângulo E9 & 90 & $20,73^{a}$ & $20,64^{a}$ & $20,52^{\mathrm{a}}$ & $20,63 \pm 1,55$ \\
\hline Ângulo G18 & 90 & $92,48^{b}$ & $92,29^{a b}$ & $98,48^{a}$ & $96,08 \pm 9,66$ \\
\hline Ângulo J16 & 90 & $93,84^{a}$ & $93,35^{\mathrm{a}}$ & $94,22^{\mathrm{a}}$ & $93,80 \pm 5,28$ \\
\hline Ângulo K19 & 90 & $75,60^{b}$ & $78,15^{\mathrm{ab}}$ & $78,94^{a}$ & $77,57 \pm 4,41$ \\
\hline Ângulo N23 & 90 & $91,29^{a}$ & $90,71^{\mathrm{a}}$ & $90,86^{a}$ & $90,95 \pm 4,30$ \\
\hline Ângulo O26 & 90 & $41,18^{a}$ & $39,83^{a}$ & $38,96^{a}$ & $39,99 \pm 4,30$ \\
\hline CAP & 90 & $5,79^{b}$ & $5,95^{\mathrm{a}}$ & $5,79^{\mathrm{b}}$ & $5,84 \pm 0,16$ \\
\hline LAP & 90 & $1,68^{\mathrm{a}}$ & $1,71^{\mathrm{a}}$ & $1,68^{\mathrm{a}}$ & $1,69 \pm 0,56$ \\
\hline DL2 & 90 & $0,30^{\mathrm{b}}$ & $0,31^{\mathrm{ab}}$ & $0,32^{\mathrm{a}}$ & $0,31 \pm 0,03$ \\
\hline DL5 & 90 & $0,20^{\mathrm{a}}$ & $0,17^{\mathrm{a}}$ & $0,15^{\mathrm{a}}$ & $0,17 \pm 0,21$ \\
\hline Ângulo W3 & 90 & $25,29^{\mathrm{a}}$ & $24,92^{\mathrm{a}}$ & $25,03^{\mathrm{a}}$ & $25,08 \pm 1,53$ \\
\hline
\end{tabular}

Médias seguidas pela mesma letra na linha não diferem pelo teste de Tukey $(p>0,05)$.

$\mathrm{CP}$ : comprimento da probóscide; $\mathrm{CF}$ : comprimento do fêmur; $\mathrm{CT}$ : comprimento da tíbia; $\mathrm{CTS}$ : comprimento do tarso; LTS: largura do tarso; CAA: comprimento da asa anterior; LAA: largura da asa anterior; Db: distância b; CAP: comprimento da asa posterior; LAP: largura da asa posterior; DL2: distância L2 e DL5: distância L5.

analisadas nas amostras oriundas dos municípios de Catolé do Rocha, Princesa Isabel e Patos. Pelo teste não foi observado diferença significativa entre os municípios de Catolé do Rocha, Princesa Isabel e Patos para as variáveis: CP; CF; Db; ângulos B4, E9, J16, N23, O26; DL5 e ângulo W3.

As medidas tomadas para as variáveis CTs, LTs e CT diferiram estatisticamente ao nível de 5\%. Essas medidas estão diretamente relacionadas à cesta de pólen das abelhas e consequentemente a sua capacidade de coleta. Segundo Souza et al. (2002), operárias de maiores áreas corbiculares são mais hábeis a levar pólen para suas colméias. A variável CTs mostrou efeito significativo $(p<0,05)$ diferindo entre o município de Catolé do Rocha e Princesa Isabel, mas as medidas tomadas nas amostras provenientes de Patos mostraram-se semelhante aos outros dois municípios. Segundo Carvalho et al. (2002b) o tipo de relevo, Borborema e Serras, encontrados na região dos municípios de Catolé do Rocha e Princesa Isabel possuem altitudes médias que variam de 500 $\mathrm{m}$ a $1010 \mathrm{~m}$, enquanto que o pediplano que caracteriza a região de Patos está em uma área deprimida entre as cristas das Serras 
com altitude média de $250 \mathrm{~m}$ acima do nível do mar. Portanto, entende-se que Patos pode ter sido um ponto de dispersão para Catolé do Rocha e Princesa Isabel através de processos enxameatórios. Entende-se que as abelhas que migraram de Patos para as localidades de Catolé do Rocha e Princesa Isabel tiveram as adaptações de comprimento do tarso em função da diferença geomorfológicas e, consequentemente, as mudanças na vegetação.

Para a variável LTS observou-se efeito significativo para os municípios de Catolé do Rocha, Princesa Isabel e Patos, porém diferindo entre Princesa Isabel e Patos. As pernas traseiras são altamente adaptadas para o transporte de pólen e própolis. A vegetação desses municípios caracterizase como plantas da caatinga arbustiva esparsa apresentando espécies produtoras de pólen e resina.

A variável ângulo G18 diferiu estatisticamente para os municípios de Patos e Catolé do Rocha, embora Princesa Isabel tenha mostrado-se igual aos outros dois municípios. O mesmo ocorreu em relação a variável K19. Para a avaliação dos ângulos G18 e K19, utiliza-se uma célula da asa ante- rior, que tem também como medida morfométrica a distância d. Dedej e Nazzi (1994) sugerem que o uso da distância d, podem ser medidos com um grau mais elevado de precisão e podem ser consideradas estimativas boas do tamanho da asa, embora não possam ser usadas para estimar o comprimento e a largura. Ruttner (1988), afirma que os ângulos das venações mostram a variação geográfica significativa, ou seja, reagem sensivelmente aos processos de evolução e podem indicar caracteres adaptativos ao meio.

Nos municípios de Catolé do Rocha e Patos a variável CAP, expressa em milímetros, mostrou efeito significativo 5,7900 e 5,7852, respectivamente, diferindo de Princesa Isabel $(5,9505)$. Considerando que, o comprimento da asa está diretamente relacionado com a distância de vôo para a coleta de alimento e considerando que embora estejam em unidades geomorfológicas semelhantes, Catolé do Rocha encontra-se em Borborema (com média $500 \mathrm{~m}$ de altitude) e Princesa Isabel em Serra (com média de 800 m de altitude), sugere-se que o comprimento de asa posterior de Princesa Isabel é uma resposta da abelha, sendo uma adaptação



Figura 1. Respresentaçao dos dados obtidos através da análise canônicas entre as cidades de Catolé do Rocha (CR), Patos (P) e Princesa Isabel (PI). (Canonic discriminate analysis plot between Catolé do Rocha (CR), Patos (P) and Princesa Isabel (PI). 
as grandes altitudes. Araújo et al. (2004) afirma que em meliponíneos a distância máxima de vôo está relacionada ao tamanho do corpo, em especial ao tamanho generalizado das asas. Para Winston (2003), as asas das abelhas são extensões do exoesqueleto adaptadas para possibilitar o vôo o que explica os valores encontrados neste estudo relacionando o tamanho das asas aos fatores adaptativos ao relevo.

Em relação à Catolé do Rocha e Patos a variável DL2 diferiu estatisticamente, porem os valores encontrados nas medidas analisadas nas amostras proveniente de Princesa Isabel $(0,3051)$ mostraram-se iguais, estatisticamente, para os municípios de Catolé do Rocha $(0,2986)$ e Patos $(0,3166)$. O que pode ser explicado pelas características geomorfológicas e pelos fatores adaptativos já citados anteriormente. Falconer (1989) encontrou uma correlação entre o genótipo e o fenótipo, que é modificada pelos efeitos ambientais.

Os dados submetidos à análise canônica estão presentes na figura 1 nos permitindo visualizar diferenças entre os municípios de Princesa Isabel (PI), Catolé do Rocha (CR) e Patos (P); ainda na figura 1 evidencia-se a existência de agrupamentos a partir dos escores das variáveis canônicas 1 e 2 (tabela II).com base nas características morfológicas, de acordo com os resultados do teste de Tocher, entre os municípios de PI e CR, mesmo havendo distância geográfica, no entanto sendo explicado em função das unidades geomorfológicas (Borboremas e Serras).

$\mathrm{Na}$ análise, através das variáveis canônicas, observa-se que a primeira e segunda variáveis canônicas detiveram aproximadamente $61 \%$ e $100 \%$ da variância, respectivamente (tabela II). A primeira variável canônica (VC1) representa um contraste entre os parâmetros CT e O26 contra K19, visto que esses parâmetros apresentam maiores coeficientes de ponderação, no entanto de sinais contrários. A segunda variável (VC2) está relacionada positivamente aos parâmetros comprimento do CTs e CAP. Apesar da distância geográfica as amostras provenientes desses municípios apresentam características morfométricas semelhantes, provavelmente, devido às condições adaptativas sofridas durante a formação dos dois grupos distintos. Embora os valores encontrados para Patos sejam próximos aos obtidos para Catolé do Rocha, os mesmos diferiram entre si ficando assim o município de Patos em outro grupo. A tabela III apresenta os resultados obtidos através do teste de Mahalanobis.

Tabela II. Variáveis canônicas obtidas a partir da análise de 20 variáveis morfométricas de abelhas Apis mellifera da mesorregião do Sertão paraibano. (Canonical variables obtained starting from the analysis of 20 variables morphometrics obtained through the analysis of bees Apis mellifera of the region of the Sertão paraibano).

\begin{tabular}{|c|c|c|}
\hline Variância & VC1 & VC2 \\
\hline Variância (autovalor) & $0,9492^{* *}$ & 0,6161 \\
\hline Coeficiente de ponderação & VC1 & VC2 \\
\hline Comprimento da probóscide & $-0,1844$ & 0,0816 \\
\hline Comprimento do fêmur & 0,4976 & $-0,3064$ \\
\hline Comprimento da tíbia & $-0,8636$ & 0,0591 \\
\hline Comprimento do tarso & 0,2188 & 0,6719 \\
\hline Largura do tarso & $-0,2065$ & $-0,1101$ \\
\hline Comprimento da asa anterior & $-0,0903$ & $-0,1028$ \\
\hline Largura da asa anterior & 0,0706 & 0,2968 \\
\hline Distância b & $-0,0341$ & $-0,0548$ \\
\hline Ângulo B4 & 0,0433 & $-0,3082$ \\
\hline Ângulo E9 & $-0,2182$ & 0,3384 \\
\hline Ângulo G18 & 0,2298 & 0,3307 \\
\hline Ângulo J16 & 0,2459 & $-0,0288$ \\
\hline & 0,5657 & $-0,0585$ \\
\hline Ângulo N23 & 0,1418 & $-0,2211$ \\
\hline Ângulo O26 & $-0,5323$ & $-0,0616$ \\
\hline Comprimento $\mathrm{C}$ & $-0,1914$ & 0,6420 \\
\hline da asa posterior & 0,2160 & $-0,0277$ \\
\hline Distância L2 & 0,4552 & $-0,2459$ \\
\hline & $-0,1259$ & $-0,1770$ \\
\hline Ângulo W3 & $-0,0714$ & $-0,2694$ \\
\hline
\end{tabular}

**significativo $(p<0,01)$ pelo teste $F$. 
Tabela III. Resultado do teste de Mahalanobis partir da análise de 20 variáveis morfométricas de abelhas Apis mellifera da mesorregião do Sertão paraibano. (Results of applied Mahalanobis test of 20 variables morfometrics obtained through the analysis of bees Apis mellifera of the region of the Sertão paraibano).

\begin{tabular}{cccc}
\hline Localidades & 1 & 2 & 3 \\
1 & 0 & $10,62^{\star *}$ & 8,15 \\
2 & $10,62^{* *}$ & 0 & $17,95^{\star *}$ \\
3 & 8,15 & $17,95^{\star *}$ & 0 \\
\hline
\end{tabular}

**significativo $(p<0,01)$ pelo teste $F$.

A entrada de abelhas no Brasil se deu com as subespécies $A$. m. mellifera, A. $m$. scutellata, A. m. caucasica, A. m. carnica e A. m. ligustica cujo cruzamento originou

\section{BIBLIOGRAFIA}

Araújo, E.D., M. Costa, J. Chaud Neto and H.G. Fowler. 2004. Body size and flight distance in stingless bees (Hymenoptera: Meliponini) Inference of flight range and possible ecological implications. Braz. J. Biol., 64: 563-568.

Carrera, M. 1980. Entomologia para você. $5^{a}$ ed. ver. e atualizada. Nobel. São Paulo.

Carvalho, M.G.R.F. de, M. do S.B. Travassos e V. da S. Maciel. 2002a. Clima, vegetação e solo. Em: Atlas escolar da Paraíba. Coordenadora: Janete Lins Rodriguez. $3^{a}$ ed. Grafset, ㄷ. João Pessoa. 112 p.

Carvalho, M.G.R.F. de, M. do S.B. Travassos e V. da S. Maciel. 2002b. Relevo e hidrografia. Em: Atlas Escolar da Paraíba/ Coordenadora: Janete Lins Rodriguez. $3^{\mathrm{a}}$ ed. Grafset, @. João Pessoa. $112 \mathrm{p}$.

Dedej, S. and F. Nazzi. 1994. Two distances of flowering vegetation as estimates of wing size. J. Apicult. Res., 33: 59-61.

Diniz-Filho, J.A.F., H.R. Hepburn, S. Radloff and S. Fuchs. 2000. Spacial analysis of morphological variantion in African honeybees (Apis mellifera L.) on continental scale. Apidologie, 31: 191204.

Falconer, D.S. 1989. Introduction to quantitative uma abelha com características próprias para cada Região, de acordo com a adaptabilidade e as mudanças geográficas existentes. Somente com a realização de estudos morfológicos e genéticos, conjuntamente, poder-se-ia estabelecer a hipótese de parentesco entre as abelhas oriundas de Catolé do Rocha e Princesa Isabel.

\section{CONCLUSÕES}

Existem dois grupos distintos morfometricamente para a mesorregião do Sertão paraibano. Há uma adaptação das abelhas, quanto às medidas morfométricas, para as diferentes regiões geomorfológicas. Sugere-se a realização de mais pesquisas comparativas entre os híbridos encontrados nessa região com o padrão das raças introduzidas. genetics. $3^{\text {rd }}$ edition. Logman. Harlow.

Ken, T., S. Fuchs, N. Koeniger and Z. Ruiguang. 2003. Morphological characterization of Apis cerana in the Yunnan Province of China. Apidologie, 34: 553-561.

Maia, G.N. 2004. Caatinga: árvores e arbustos e suas utilidades. 1 ed. D\&Z Computação Gráfica e Editora. São Paulo. 413 p.

Ruttner, F. 1988. Biogeography and taxonomy of honeybees. Springer-Verlag. Berlin. $248 \mathrm{p}$.

SAEG. 1999. Versão 5.0. Fundação Arthur Bernardes. Universidade Federal de Viçosa. Viçosa, MG.

Sereno, F.T.P.S., F.A. Padilla, D. Message, E. Vilela e J.R.B. Sereno. Diferenças morfométricas entre colmeias de três regiões brasileiras. In: Reunião Anual da Sociedade Brasileira de Zootecnia, 38, Recife, PE. Anais. SBZ. Recife.

Souza, D.C., C.D. Cruz, L.A. de O. Campos and A.J. Regazzi. 2002. Correlation between honey production and some morphological traits in africanized honey bees (Apis mellifera). Ciência Rural, 32: 869-872.

Winston, M.L. 2003. A biologia da abelha. Tradução de Carlos A. Osowski. Magister. Porto Alegre. 276 p.

Archivos de zootecnia vol. 58, núm. 221, p. 71. 